\title{
Prediction of Adrenocortical Insufficiency After Pituitary Adenoma Surgery Using Postoperative Basal Cortisol Levels
}

\author{
V. HÁNA Jr. ${ }^{1}$, J. JEŽKOVÁ ${ }^{1}$, M. KOSÁK ${ }^{1}$, M. KRŠEK ${ }^{1}$, J. MAREK $^{1}$, D. NETUKA ${ }^{2}$, \\ M. HILL ${ }^{3}$, V. HÁNA ${ }^{1}$ \\ ${ }^{1}$ Third Department of Internal Medicine, General University Hospital and First Faculty of Medicine, \\ Charles University, Prague, Czech Republic, ${ }^{2}$ Department of Neurosurgery, Central Military \\ Hospital, First Faculty of Medicine, Charles University, Prague, Czech Republic, ${ }^{3}$ Steroid Hormone \\ Unit, Institute of Endocrinology, Prague, Czech Republic
}

Received July 20, 2014

Accepted October 8, 2014

On-line December 3, 2014

\begin{abstract}
Summary
Our aim was to analyze the correlation of early postoperative cortisol levels in patients after transsphenoidal pituitary adenoma surgery compared to the standard dose ACTH test and Insulin tolerance test (ITT) several months later. We retrospectively reviewed data from 94 patients operated for pituitary adenoma in years 2009-2012. The comparison of day 7 (median) postoperative basal cortisol levels and 3.6 months (median) after pituitary adenoma surgery stimulation test - standard dose $250 \mu \mathrm{g} 1-24 \mathrm{ACTH}$ test in 83 patients or ITT in 11 patients were performed. All 16 patients with early postoperative cortisol levels $>500 \mathrm{nmol} / \mathrm{l}$ proved a sufficient response in the stimulation tests. At basal cortisol levels of $370-500 \mathrm{nmol} / \mathrm{l}$ the sufficient response was found in $96 \%(27 / 28)$ of patients. In the postoperative basal cortisol levels $200-370 \mathrm{nmol} / \mathrm{l}$ we found a preserved corticotroph axis later on in $88 \%(28 / 32)$ of cases. Patients with basal cortisol levels $100-200 \mathrm{nmol} / \mathrm{l}$ had a maintained corticotroph axis function in $8 / 11$ cases $-73 \%$. All patients with an early postoperative basal cortisol level above $500 \mathrm{nmol} / \mathrm{l}$ proved in the stimulation tests a preserved corticotroph axis function. The interval $370-500 \mathrm{nmol} / \mathrm{l}$ showed a minimal risk of postoperative adrenal insufficiency.
\end{abstract}

\section{Key words}

Adrenal insufficiency - Hypocorticalism - Pituitary adenoma • Surgery $\bullet$ ACTH test

\begin{abstract}
Corresponding author
V. Hána Jr., Third Department of Internal Medicine, General University Hospital and First Faculty of Medicine, Charles University, U Nemocnice 1, 12808 Prague 2, Czech Republic. Fax: 00420224919780 . E-mail: vaczech@gmail.com
\end{abstract}

\section{Introduction}

One of the key procedures in the postoperative care for patients after a pituitary tumor surgery is the decision to continue with glucocorticoid replacement therapy. There have been consistent data showing that patients with basal cortisol levels under $100 \mathrm{nmol} / \mathrm{l}$ show up as glucocorticoid insufficient with a need for lifelong glucocorticoid replacement. On the other hand patients with basal cortisol levels above $500 \mathrm{nmol} / 1$ (400-500 from different authors) prove in stimulation tests a preserved hypothalamo-pituitary-adrenal (HPA) axis function (Courtney et al. 2000, Klose et al. 2005, Jayasena et al. 2009). The remaining group of patients with postoperative cortisol levels between 100 and 400$500 \mathrm{nmol} / \mathrm{l}$ has to be evaluated.

Many possible tests have been advised to assess the postoperative adrenal function. An Insulin tolerance test (ITT) has long been considered as a gold standard for testing HPA axis function, but a more practical alternative, a short ACTH test is getting more popular for its simplicity, fewer contraindications, price (in countries with a high staff labor price). The ACTH test has also 
shown a good correlation with the ITT (Stewart et al. 1988). There is still a debate about the dose of ACTH used with reasonable arguments for both alternatives 1 and $250 \mu \mathrm{g}$ (Agha et al. 2006, Abdu et al. 1999, Tordjman et al. 2000). Other tests such as metyrapone or glucagon tests were also tested for the diagnosis of secondary adrenal insufficiency, but do not play a major role in most of the endocrinology departments (Inder and Hunt 2002). Other methods for postoperative HPA axis function evaluation have been suggested such as pre- and postoperative basal cortisol index (Zada et al. 2012) or DHEA, DHEA-S levels after a low dose ACTH test (Sayyed Kassem et al. 2012).

Concerning the time of definitive testing it has been shown that using the ACTH test, HPA axis can be reliably tested from 1-3 months postoperatively (Klose et al. 2005, Inder and Hunt 2002). Up to this time the ITT, metyrapone or glucagon tests are proposed as the test of choice. In some patients due to an early testing a HPA axis recovery in later period could be underestimated (Inder and Hunt 2002).

\section{Patients and Methods}

From all patients with pituitary adenomas operated between 1.1.2009 - 31.12.2012 in the Dept. of Neurosurgery, Central Military Hospital, $1^{\text {st }}$ Medical Faculty, Charles University and treated in the $3^{\text {rd }}$ Dept. of Internal Medicine, General University Hospital in Prague medical records were retrospectively analyzed. A group of 94 patients (Table 1), where a postoperative HPA axis stimulation test - ACTH test or ITT was performed, was selected and analyzed. This group included patients with non-functioning pituitary adenomas, GH producing adenomas, prolactinomas. Very few patients with basal cortisol repeatedly below $100 \mathrm{nmol} / \mathrm{l}$ or above $500 \mathrm{nmol} / \mathrm{l}$ were included in the analysis, because in these groups the stimulation test is not routinely performed and so we included only several, where we did the stimulation testing. Besides the patients with Cushing disease, also those who were reoperated were excluded. The experiments comply with the current laws of the country in which they were performed.

Table 1. Group characteristics.

\begin{tabular}{lcccc}
\hline & Total & NF & GH & PRL \\
\hline$n$ & 94 & 61 & 29 & 4 \\
Age (years) & $53.3 \pm 30.2$ & $57.7 \pm 28.8$ & $46.2 \pm 26.8$ & $36.2 \pm 10.6$ \\
Men/Women & $49 / 45$ & $31 / 30$ & $16 / 13$ & $2 / 2$ \\
Postoperative adrenal & 13 & 12 & 0 & 1 \\
insufficiency & & & & \\
\hline
\end{tabular}

$\mathrm{NF}$ - non-functioning adenoma, GH - growth hormone producing adenoma, PRL - prolactinoma; age shown as mean $\pm 2 \mathrm{SD}$

HPA axis is not routinely preoperatively evaluated by dynamic testing, because pituitary function is expected to be changed by operation and patients are covered during the pituitary surgery and postoperatively with corticosteroids anyway. Only those with very low morning cortisol levels and/or with clinical symptoms of hypocortisolism are preoperatively replaced with hydrocortisone without dynamic testing of HPA axis. Under such conditions 9 patients were preoperatively replaced with hydrocortisone. Based on low basal free T4 levels and inappropriately low TSH had preoperatively 22/94 patients secondary hypothyroidism. Secondary hypogonadism (using total testosterone and gonadotropins) was diagnosed preoperatively in 31/49 males; premenopausal women were hypogonadal in 6 out of 15 (evaluation of gonadal axis in postmenopausal women is not accurate). Patients had an adequate replacement with thyroid hormone pre and postoperatively, if necessary. None of the patients received contraceptives or estrogen substitution therapy during the analyzed period.

All patients underwent an endonasal endoscopic adenomectomy with a standard postoperative hydrocortisone replacement. After the operation a hormonal testing was done including basal cortisol level on day $7(6,8$; expressed as median with quartiles) at 7 am, with a withdrawal of hydrocortisone replacement at least $13 \mathrm{~h}$ before the blood test was done. In patients with repeated basal cortisol level $>500 \mathrm{nmol} / \mathrm{l}$ the hydrocortisone treatment was omitted, in the rest the 
hydrocortisone replacement was continued in a replacement dose $15-20 \mathrm{mg}$ /day with an increase during stress and exercise up to the definitive testing a few months later. All subjects were followed in an outpatient department and their adrenocortical function was tested after 3.6 months $(64,220$ days; expressed as median with quartiles). For the HPA axis evaluation a standard $250 \mu \mathrm{g}$ dose ACTH test in 83 patients and ITT in 11 patients was performed.

Using the ACTH test a $250 \mu \mathrm{g} 1-24 \mathrm{ACTH}$ (Synacthen $^{\circledR}$ ) was administered intravenously with a 30 and $60 \mathrm{~min}$ plasma cortisol measurement. In the evaluation of sufficiency the higher value of these two was used. In the ITT a dose of 0.1-0.2 IU/ $\mathrm{kg}$ rapid acting insulin was administered intravenously and hypoglycemia $<2.2 \mathrm{mmol} / 1$ was reached. 30, 60, $90 \mathrm{~min}$ samples were taken. The highest cortisol level was used. In both tests a cortisol cut-off level of $500 \mathrm{nmol} / 1$ after stimulation was set as a level for HPA axis sufficiency.

Analytical method used for cortisol measurement was ADVIA Centaur Cortisol assay - a competitive immunoassay using direct chemiluminescent method. Interassay $\mathrm{CV}=3.07 \%$, intraassay $\mathrm{CV}=$ $2.89 \%$. Analytical sensitivity $=27.6 \mathrm{nmol} / 1$.

The differences between basal and stimulated cortisol levels for ITT and ACTH tests were evaluated using a repeated measures ANOVA model consisting of subject factor separating inter-individual variability, between-subject Factor test (ITT vs. ACTH test), withinsubject factor Stimulation (basal vs. stimulation) and Factor $\times$ Stimulation interaction. The original data were transformed to attain symmetry and constant variance by a Box-Cox transformation.

\section{Results}

All 16 patients with early postoperative cortisol levels $>500 \mathrm{nmol} / \mathrm{l}$ proved a sufficient response in the stimulation tests. 78 out of 94 patients had a postoperative basal cortisol levels $<500 \mathrm{nmol} / \mathrm{l}$ (Fig. 1). From these 78 patients only 13 patients $(17 \%)$ showed insufficient response $(<500 \mathrm{nmol} / \mathrm{l})$ during the stimulation test a few months later. 10 patients were tested by the short ACTH test and 3 patients by ITT.

In the group with basal cortisol levels $<100 \mathrm{nmol} / \mathrm{l}$ only 2 out of 7 patients had a sufficient HPA axis function after the stimulation (all had ACTH test, with insufficient maximal stimulated cortisol levels 202, $278,386,447,480 \mathrm{nmol} / 1$, respectively).
With basal cortisol levels 100-200 nmol/1 a maintained HPA axis function was demonstrated in 8 out of 11 patients - $73 \%$ (6/8 in ACTH test and 2/3 in ITT). The insufficient maximal cortisol responses after the stimulation were 190, 366, $469 \mathrm{nmol} / 1$, respectively.

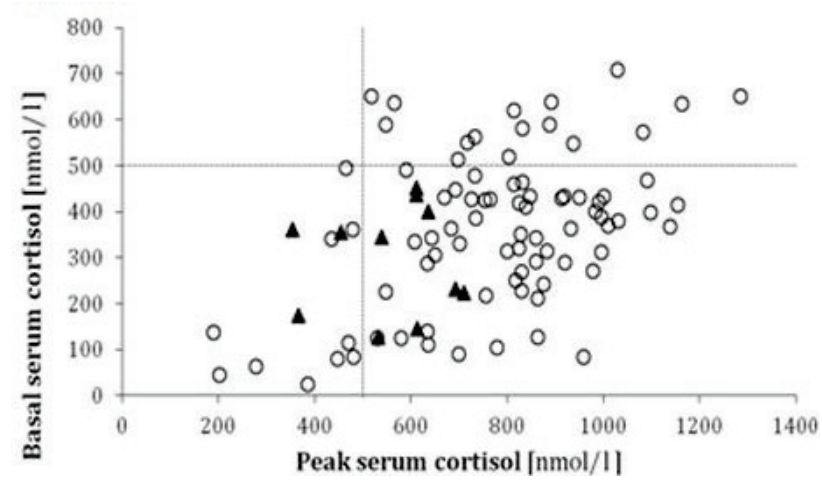

Fig. 1. Postoperative basal cortisol level (median day 7, range 68) vs. peak serum cortisol in the stimulation test (median 3.6 months after surgery). o-ACTH test, $\mathbf{\Delta}-$ Insulin tolerance test

Then a group of patients with basal cortisol levels 200-370 nmol/1 was selected due to the distribution of the results (Fig. 1). In this cohort $88 \%$ of patients had a sufficient HPA axis testing (25/27 tested by ACTH test and $3 / 5$ by the ITT) with insufficient maximal stimulated cortisol values: $354,435,454,479 \mathrm{nmol} / 1$, respectively.

In the group with basal cortisol levels 370-500 nmol/1 27/28 patients - $96 \%$ had sufficient response (24/25 in the ACTH test, $3 / 3$ in ITT). The only one patient had a basal cortisol level $494 \mathrm{nmol} / \mathrm{l}$ and maximal response of $465 \mathrm{nmol} / 1$ (60 $\mathrm{min}$ ) in the ACTH test.

From the 9 patients with corticosteroid replacement preoperatively 7 had normal HPA axis function postoperatively and 2 had adrenal insufficiency after the operation. Apart from these, there were 11 patients with newly diagnosed adrenal insufficiency after the operation.

The ANOVA model demonstrated significant differences between basal and stimulated values as expected and significantly higher maximum levels in ACTH test when compared with ITT.

\section{Discussion}

In the postoperative period after pituitary surgery an evaluation of pituitary axes plays an important role. The key and potentially life saving is the management of the HPA axis function. From the clinicians' point of view, a basal cortisol level is the 
earliest and only measure to assess the HPA axis function in the early postoperative period. It has neither high specificity nor sensitivity for the diagnosis of adrenal insufficiency in all patients, but showed a good prognostic value in the marginal groups.

It has been repeatedly noted that basal cortisol levels postoperatively $<80-111 \mathrm{nmol} / \mathrm{l}$ are highly indicative of adrenal insufficiency (Courtney et al. 2000, Jayasena et al. 2009, Inder and Hunt 2002, Karaca et al. 2010, Watts and Tindall 1988). On the other side of scale a sufficient level of basal cortisol permitting a safe withdrawal of hydrocortisone substitution showed more variation, $450 \mathrm{nmol} / 1$ (Courtney et al. 2000, Inder and Hunt 2002), $400 \mathrm{nmol} / 1$ (Klose et al. 2005), $392 \mathrm{nmol} / 1$ (Jayasena et al. 2009). In our conditions the basal cortisol level with $100 \%$ specificity for detecting only adrenal insufficient patients is $82 \mathrm{nmol} / \mathrm{l}$, whereas $100 \%$ specificity for adrenal sufficiency would be $495 \mathrm{nmol} / \mathrm{l}$. This higher result compared with other publications is distorted by only one patient with basal cortisol $494 \mathrm{nmol} / 1$ and stimulated $465 \mathrm{nmol} / 1$. Without this exceptional patient a more statistically powerful cut-off level of basal cortisol would be $370 \mathrm{nmol} / \mathrm{l}$ under which stand all the other subjects with adrenal insufficiency and which would be more in agreement with previous works. In these two groups with highly probable HPA axis insufficiency and sufficiency respectively, no further stimulation tests would technically be necessary in most of these patients. This cannot be applied absolutely, because rarely a delayed adrenal insufficiency was published (Gleeson et al. 2003).

The remaining group with uncertain basal cortisol levels needs to have a stimulation test. Some authors divide this group in two subgroups according to the need of permanent glucocorticoid substitution or only stress period glucocorticoid administration up to the definitive HPA axis testing (Courtney et al. 2000, Khan et al. 2011). They suggest a safe level for withdrawal of permanent corticosteroid replacement early after surgery when a basal cortisol level is higher than 250 , or 276 nmol/1, respectively (Courtney et al. 2000, Khan et al. 2011). Using such criteria Khan et al. (2011) presented in their cohort, that HPA axis insufficiency in the dynamic testing was not uncommon in a group of patients with "sufficient" postoperative day-3 basal cortisol level (11 out of 46 patients). Nevertheless only two of these patients had long term regular cortisol replacement (Khan et al. 2011). The subgroup of patients without replacement and finally with proved adrenocortical insufficiency might be from our point of view endangered by hypocortical state in a case of serious stress (infection, trauma, surgery etc.). We prefer to use the glucocorticoid replacement in all of these patients with the aim to minimalize the risk of clinically serious hypocorticalism, but we use lower replacement doses than mentioned in the study of Khan et al. (2011), where authors used supraphysiological doses of hydrocortisone $30 \mathrm{mg}$ daily in a significant number of patients. Our clinical experience is that using currently usual replacement doses 15-20 mg of hydrocortisone per day, the dosage based on pharmacokinetic studies (Mah et al. 2004), does not cause any side effects when used for a short period till the stimulation test.

The ACTH test has shown a good correlation with the ITT (Stewart et al. 1988). However, in the postoperative time the ACTH test could underestimate the adrenal insufficiency due to a maintained sensitivity of adrenal cortex to direct adrenal stimulation in the ACTH test shortly after a pituitary insult. Thus the definitive testing should not be done earlier than 1-3 months postoperatively (Klose et al. 2005, Inder and Hunt 2002). In our study a part of the group had an ITT but from the statistical point of view the group was too small to make a statistically significant conclusion only from the ITT. The majority of the patients had an ACTH test because of its simplicity, low limitations, good accessibility of 1-24ACTH. We used a $250 \mu \mathrm{g}$ ACTH stimulation test, which is widely accepted and its utility was confirmed in many studies (Agha et al. 2006, Abdu et al. 1999, Tordjman et al. 2000). This test avoids the risk of error after an inaccurate dilution, which is stressed by critics of the $1 \mu \mathrm{g}$ ACTH test. We used a cut off level for the ACTH test of $500 \mathrm{nmol} / 1$ which is in correlation with other published studies and recommended cut off levels and in our experience reflects the clinical outcome in our patients. The higher stimulated cortisol levels for ACTH test described by the statistical analysis may be ascribed to the non-randomized selection of patients for each test, where ITT has several limitations as described above.

In conclusion a basal cortisol level measurement reliably predicts a maintained HPA axis function in all patients with cortisol levels above $500 \mathrm{nmol} / \mathrm{l}$ allowing a withdrawal of hydrocortisone replacement therapy. In patients with cortisol levels $370-500 \mathrm{nmol} / \mathrm{l}$ there is a very high probability of maintained HPA axis function. In this group in clinically selected cases the regular continuation of hydrocortisone replacement probably might be used 
only in stressful situations and the later stimulation testing is necessary. Basal cortisol levels under $370 \mathrm{nmol} / 1$ require a hydrocortisone treatment up to the definitive testing a few months later. In patients with basal cortisol under 100 $\mathrm{nmol} / \mathrm{l}$ postoperatively there is a high probability of adrenal insufficiency subsequently, but in individual cases a restitution of HPA axis can appear.

\section{Conflict of Interest}

There is no conflict of interest.

\section{Acknowledgements}

This project was supported by the Research project of Charles University (Prvouk, P25/LF1/2).

\section{References}

ABDU TA, ELHADD TA, NEARY R, CLAYTON RN: Comparison of the low dose short synacthen test (1 microg), the conventional dose short synacthen test (250 microg), and the insulin tolerance test for assessment of the hypothalamo-pituitary-adrenal axis in patients with pituitary disease. J Clin Endocrinol Metab 84: 838-843, 1999.

AGHA A, TOMLINSON JW, CLARK PM, HOLDER G, STEWART PM: The long-term predictive accuracy of the short synacthen (corticotropin) stimulation test for assessment of the hypothalamic-pituitary-adrenal axis. J Clin Endocrinol Metab 91: 43-47, 2006.

COURTNEY CH, MCALLISTER AS, MCCANCE DR, BELL PM, HADDEN DR, LESLIE H, SHERIDAN B, ATKINSON AB: Comparison of one week $0900 \mathrm{~h}$ serum cortisol, low and standard dose synacthen tests with a 4 to 6 week insulin hypoglycaemia test after pituitary surgery in assessing HPA axis. Clin Endocrinol (Oxf) 53: 431-436, 2000.

GLEESON HK, WALKER BR, SECKL JR, PADFIELD PL: Ten years on: Safety of short synacthen tests in assessing adrenocorticotropin deficiency in clinical practice. J Clin Endocrinol Metab 88: 2106-2111, 2003.

INDER WJ, HUNT PJ: Glucocorticoid replacement in pituitary surgery: guidelines for perioperative assessment and management. J Clin Endocrinol Metab 87: 2745-2750, 2002.

JAYASENA CN, GADHVI KA, GOHEL B, MARTIN NM, MENDOZA N, MEERAN K, DHILLO WS: Day 5 morning serum cortisol predicts hypothalamic-pituitary-adrenal function after transsphenoidal surgery for pituitary tumors. Clin Chem 55: 972-977, 2009.

KARACA Z, TANRIVERDI F, ATMACA H, GOKCE C, ELBUKEN G, SELCUKLU A, UNLUHIZARCI K, KELESTIMUR F: Can basal cortisol measurement be an alternative to the insulin tolerance test in the assessment of the hypothalamic-pituitary-adrenal axis before and after pituitary surgery? Eur $J$ Endocrinol 163: 377-382, 2010.

KHAN MI, HABRA MA, MCCUTCHEON IE, NOGUERAS-GONZALES GM, DEVIN JK, BUSAIDY NL, LEVINE NB, LINDSTROM W, KAGAN D, JIMENEZ C, WAGUESPACK SG: Random postoperative day-3 cortisol concentration as a predictor of hypothalamic-pituitary-adrenal axis integrity after transsphenoidal surgery. Endocr Pract 17: 717-726, 2011.

KLOSE M, LANGE M, KOSTELJANETZ M, POULSGAARD L, FELDT-RASMUSSEN U: Adrenocortical insufficiency after pituitary surgery: an audit of the reliability of the conventional short synacthen test. Clin Endocrinol (Oxf) 63: 499-505, 2005.

MAH PM, JENKINS RC, ROSTAMI-HODJEGAN A, NEWELL-PRICE J, DOANE A, IBBOTSON V, TUCKER GT, ROSS RJ: Weight-related dosing, timing and monitoring hydrocortisone replacement therapy in patients with adrenal insufficiency. Clin Endocrinol (Oxf) 61: 367-375, 2004.

SAYYED KASSEM L, EL SIBAI K, CHAIBAN J, ABDELMANNAN D, ARAFAH BM: Measurements of serum DHEA and DHEA sulphate levels improve the accuracy of the low-dose cosyntropin test in the diagnosis of central adrenal insufficiency. J Clin Endocrinol Metab 97: 3655-3662, 2012.

STEWART PM, CORRIE J, SECKL JR, EDWARDS CR, PADFIELD PL: A rational approach for assessing the hypothalamo-pituitary-adrenal axis. Lancet 1: 1208-1210, 1988. 
TORDJMAN K, JAFFE A, TROSTANETSKY Y, GREENMAN Y, LIMOR R, STERN N: Low-dose (1 microgram) adrenocorticotrophin (ACTH) stimulation as a screening test for impaired hypothalamo-pituitary-adrenal axis function: sensitivity, specificity and accuracy in comparison with the high-dose (250 microgram) test. Clin Endocrinol (Oxf) 52: 633-640, 2000.

WATTS NB, TINDALL GT: Rapid assessment of corticotropin reserve after pituitary surgery. JAMA 259: 708-711, 1988.

ZADA G, TIROSH A, HUANG AP, LAWS ER, WOODMANSEE WW: The postoperative cortisol stress response following transsphenoidal pituitary surgery: a potential screening method for assessing preserved pituitary function. Pituitary 16: 319-325, 2013. 